\title{
The selectivity of aversive memory reconsolidation and extinction processes depends on the initial encoding of the Pavlovian association
}

\author{
Jacek Debiec, ${ }^{1,2,3,7,8}$ Lorenzo Diaz-Mataix, ${ }^{1,7}$ David E.A. Bush, ${ }^{1}$ Valérie Doyère, ${ }^{1,4,5}$ \\ and Joseph E. LeDoux ${ }^{1,6}$ \\ ${ }^{1}$ W.M. Keck Foundation Laboratory of Neurobiology, Center for Neural Science, New York University, New York 10003, USA; \\ ${ }^{2}$ Molecular \& Behavioral Neuroscience Institute, University of Michigan, Ann Arbor, Michigan 48109, USA; ${ }^{3}$ Department \\ of Psychiatry, University of Michigan, Ann Arbor, Michigan 48109, USA; ${ }^{4}$ Université Paris-Sud, Centre de Neurosciences \\ Paris-Sud, Orsay F-91405, France; ${ }^{5}$ Centre National de la Recherche Scientifique, Orsay F-91405, France; ${ }^{6}$ The Emotional \\ Brain Institute, Nathan Kline Institute for Psychiatric Research, Orangeburg, New York 10962, USA
}

In reconsolidation studies, memories are typically retrieved by an exposure to a single conditioned stimulus (CS). We have previously demonstrated that reconsolidation processes are CS-selective, suggesting that memories retrieved by the CS exposure are discrete and reconsolidate separately. Here, using a compound stimulus in which two distinct CSs are concomitantly paired with the same aversive unconditioned stimulus (US), we show in rats that reexposure to one of the components of the compound CS triggers extinction or reconsolidation of the other component. This suggests that the original training conditions play a critical role in memory retrieval and reconsolidation.

In Pavlovian threat (fear) conditioning, a neutral conditioned stimulus (CS), such as a tone or a light, is paired with an aversive unconditioned stimulus (US), typically a mild electric shock (LeDoux 2012). Neurons in the lateral nucleus of the amygdala (LA) play a critical role in memory acquisition, consolidation, storage, retrieval, reconsolidation, and extinction (Sotres-Bayon et al. 2009; Johansen et al. 2011; Maren 2011).

Retrieval of the memory through an exposure to the CS allows memory modification and/or restorage (Tronson and Taylor 2007; Finnie and Nader 2012). If a single cue (CS or US) or a single CS-US pairing is presented, memories may be enhanced, attenuated, or updated through reconsolidation processes (Lee 2009; Debiec et al. 2011; Dudai 2012; Díaz-Mataix et al. 2013; Stern and Alberini 2013), whereas multiple presentations of the same CS trigger extinction processes which are believed to reduce the conditioned response through a new learning without changing the original memory trace (Myers and Davis 2007; Pérez-Cuesta and Maldonado 2009; Maren 2011; Milad and Quirk 2012). It has been proposed that whether a retrieved memory undergoes reconsolidation or extinction processes is dependent upon the dominance of the particular (original or new) memory trace at the time of retrieval (Eisenberg et al. 2003; Runyan and Dash 2005).

Using Pavlovian threat conditioning in rats and pharmacological manipulations in the LA, we have previously shown that reconsolidation processes are stimulus selective (Debiec et al. 2006, 2010; Doyère et al. 2007; Diaz-Mataix et al. 2011). In one of these studies, we used a dual auditory threat conditioning protocol in which two distinct acoustic CSs were each paired in separate trials with the same electric footshock US (Doyère et al.

\footnotetext{
${ }^{7}$ These authors equally contributed to this work.

${ }^{8}$ Corresponding author

E-mail jdebiec@umich.edu

Article is online at http://www.learnmem.org/cgi/doi/10.1101/lm.031609.113.
}

2007). Reexposure to one of the CSs followed by intra-LA disruption of reconsolidation processes with a protein synthesis inhibitor, anisomycin, impaired responding to the presented CS but not to the nonreactivated one, suggesting that memories retrieved by the CS are distinct and reconsolidate separately.

In the majority of reconsolidation studies so far, memories are typically reactivated by an exposure to a single CS, whether it is a simple stimulus (tone or light) or a complex conditioning context. However, in real life an organism is simultaneously exposed to various stimuli from different modalities. The question arises whether distinct events, such as tone and light, presented together during training reconsolidate together. In other words, will an exposure to a single component of a learning experience involving distinct modalities render the other component labile?

To address this issue, we trained rats in a Pavlovian threat conditioning paradigm with a compound (tone + light $[\mathrm{T}+\mathrm{L}]$ ) stimulus as a CS, and tested whether reactivating the memory by presenting the light CS will trigger the reconsolidation of the tone memory. Adult Sprague-Dawley male rats weighing around $300 \mathrm{~g}$ (Hiltop Laboratories, Scottdale, PA) were housed individually in plastic Nalgene cages and maintained on a 12-h light-dark cycle, with food and water provided ad libitum. All procedures were in accordance with the NIH Guide for the Care and Use of Experimental Animals, and were approved by the New York University Animal Care and Use Committee. Under Nembutal anesthesia $(45 \mathrm{mg} / \mathrm{kg}$, i.p.), rats were implanted bilaterally with 22-gauge stainless-steel guide cannulae aimed at the lateral nuclei of the amygdala (LA, AP $3.0 \mathrm{~mm}, \mathrm{~L} \pm 5.3 \mathrm{~mm}, \mathrm{DV}-8.0 \mathrm{~mm}$ from

(C) 2013 Debiec et al. This article is distributed exclusively by Cold Spring Harbor Laboratory Press for the first 12 months after the full-issue publication date (see http://learnmem.cshlp.org/site/misc/terms.xhtml). After 12 months, it is available under a Creative Commons License (AttributionNonCommercial 3.0 Unported), as described at http://creativecommons. org/licenses/by-nc/3.0/. 
A

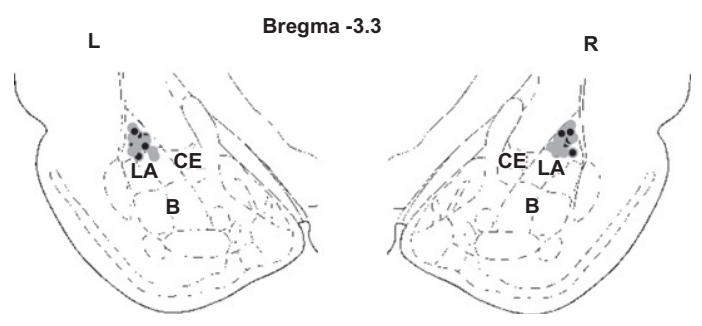

B
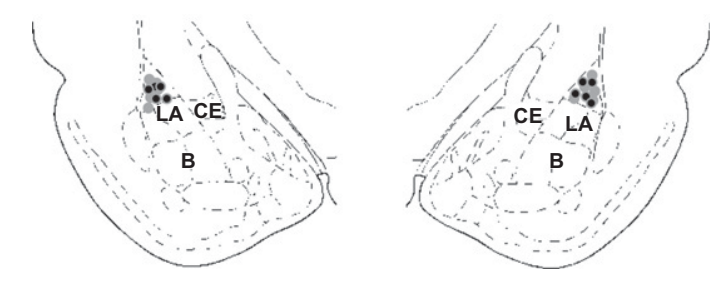

C
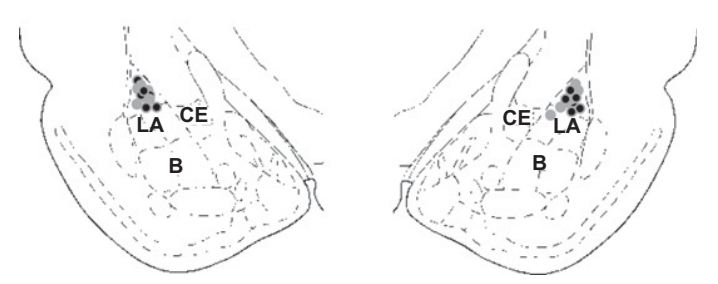

Figure 1. Cannulae placements in the LA. Images show placements of the injector cannula tip in experiments described in Figure $2 \mathrm{~A}(A)$, Figure $2 \mathrm{~B}(B)$, and Figure $2 \mathrm{C}(C)$. All sections depicted at around $3.3 \mathrm{~mm}$ posterior to bregma. Gray filled circles indicate ACSF groups; black filled circles indicate anisomycin groups. (B) Basal nucleus of the amygdala, (CE) central nucleus of the amygdala, (LA) lateral nucleus of the amygdala.

the skull surface [Paxinos and Watson 1986]). The guide cannulae were fixed to the skull using acrylic dental cement. A dummy cannula was inserted into each guide cannula to prevent clogging. At the end of each behavioral experiment, rats were anesthetized with an overdose of chloral hydrate $(600 \mathrm{mg} / \mathrm{kg})$ and perfused with $10 \%$ buffered formalin. Nissl staining and light microscopy were used to verify the location of injector cannula tips in the LA (Fig. 1). All training procedures were conducted in Context A; for all other procedures Context B was used. Context A included an odorless Plexiglas chamber with transparent walls, rectangular in shape, at a horizontal plane and a metal grid floor (Model E10-10). The chamber was dimly illuminated by a red light and enclosed within a sound attenuating chamber (Model E10-20). Context B was a Plexiglas chamber (Model E10-10) with a custommade cream-colored Plexiglas wall insert, triangular in shape, at the horizontal plane. The floor was covered with a smooth black Plexiglas floor. In addition, $0.05 \mathrm{~mL}$ isoamyl acetate (Sigma Aldrich) was placed on the external side of the insert wall.

After at least $7 \mathrm{~d}$ recovery from the surgery, rats were habituated to Context $\mathrm{A}$ for $2 \mathrm{~d}$ (1-h sessions). On day 3 the rats were trained using a compound stimulus conditioning protocol [ $(\mathrm{T}+$ L)-US] involving an auditory (20-sec tone, $5 \mathrm{kHz}, 80 \mathrm{~dB}$ ) and a visual (20-sec white light) component presented simultaneously and co-terminating with a footshock US $(0.8 \mathrm{~mA}, 0.5 \mathrm{sec})$ (see Fig. 2A, top, for the schematic of behavioral procedures). Four training trials were given; the inter-trial interval (ITI) was random- ly generated and was 1-4 min. Twenty-four hours later, rats were placed in Context B. Following 2 min of acclimation, rats were exposed to a single light (L) presentation $(20 \mathrm{sec})$. Freezing during the $\mathrm{L}$ stimulus was measured and used to equate performance for groups that were to receive either drug or vehicle. Immediately
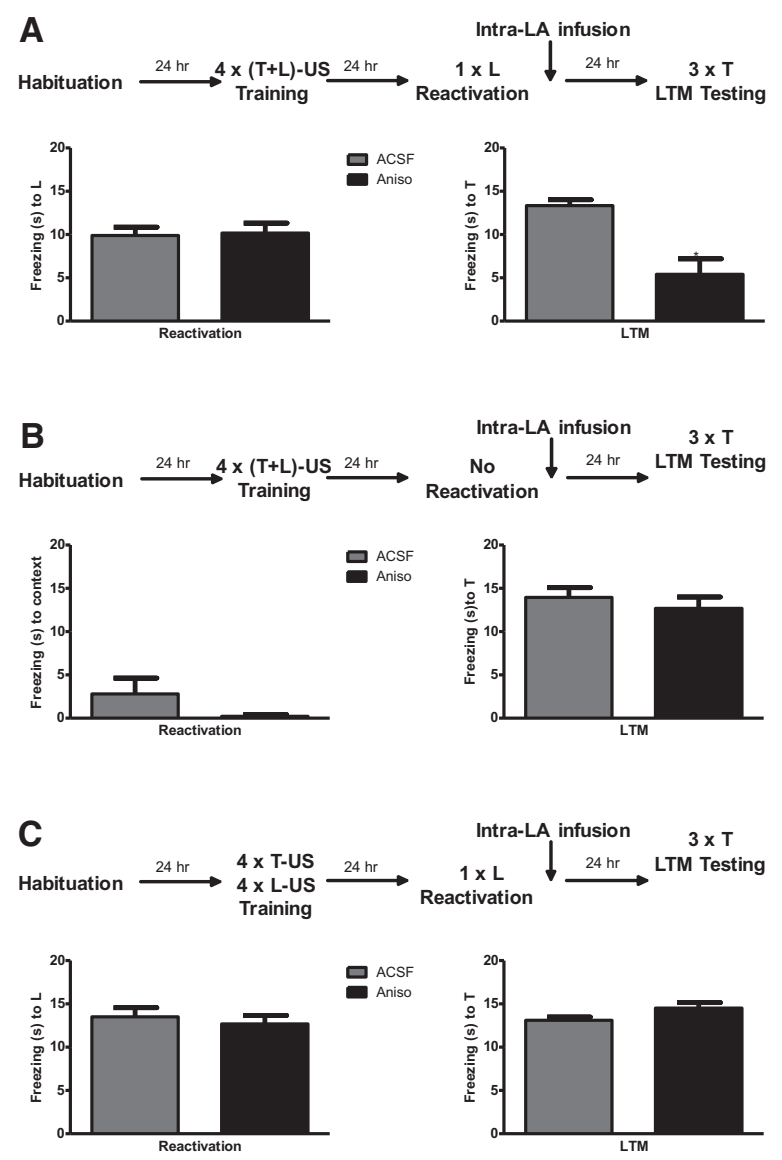

Figure 2. Retrieval of one of the components of the compound stimulus triggers in the LA protein synthesis-dependent memory reconsolidation of the other component. (A) Rats trained using a compound stimulus (light + tone) conditioning procedure and infused with anisomycin in the LA after exposure to the light component of the compound show decreased freezing to the tone during long-term memory (LTM) test. Top panel: chart showing the experimental design. Left graph: mean \pm SEM freezing in seconds of rats treated with ACSF or anisomycin in response to the light exposure during reactivation. Right graph: mean \pm SEM freezing in response to the tone averaged across three tone presentations during LTM. (B) Rats trained with a compound stimulus and infused with anisomycin in the LA with no reactivation of memory do not show any loss of freezing during LTM. Top panel: chart showing the experimental design. Left graph: contextual freezing in seconds (mean \pm SEM) of rats treated with ACSF or anisomycin during context exposure. Right graph: mean \pm SEM freezing in response to the tone averaged across three presentations during LTM. (C) When the same stimuli (light and tone) are not presented during training as a compound, but are paired separately with the unconditioned stimulus (light-US and tone-US pairings, respectively), the intra-LA infusion of anisomycin after the retrieval of the light does not affect the freezing to the tone during LTM. Top panel: chart showing the experimental design. Left graph: freezing to the light (mean \pm SEM) of rats treated with ACSF or anisomycin during memory reactivation. Right graph: mean \pm SEM freezing in response to the tone averaged across tone presentations during LTM. (T) Tone, (L) light, (US) unconditioned stimulus, (LTM) long-term memory, (ACSF) artificial cerebrospinal fluid, (Aniso) anisomycin, $\left(^{*}\right) P$ $<0.05$ vs. ACSF group. 
after, rats were infused into the LA with either anisomycin $(n=6$, $62.5 \mu \mathrm{g} / 0.5 \mu \mathrm{L}, 0.2 \mu \mathrm{L} / 2 \mathrm{~min} /$ side; Sigma-Aldrich) or artificial cerebrospinal fluid (ACSF) $(n=8)$. Twenty-four hours later, in Context $\mathrm{B}$, animals were given three tone $(\mathrm{T})$ alone presentations for assessing their long-term memory (LTM) to the T element of the compound. Behavior during LTM tests was videotaped and freezing responses to the tone presentations were scored off-line by an observer blind to the experimental conditions. An average of the three scores for each tone for each rat was used for the statistical analysis.

The results show that while freezing to the $\mathrm{L}$ stimulus during retrieval was equivalent in both groups $\left(t_{(12)}=0.195\right.$, n.s.), rats infused with anisomycin showed significantly less freezing to the tone in the LTM test than ACSF-infused rats $\left(t_{(12)}=4.620, P<\right.$ 0.001 ) (Fig. 2A). In contrast, when no stimulus was presented during the reactivation phase ( $2 \mathrm{~min}$ and 20 -sec exposure to Context B; see Fig. 2B, top, for an outline of the procedure), both ACSF ( $n=$ $5)$ and Aniso $(n=5)$ groups showed a very low level of freezing to the context during the reactivation phase (unequal variance $t_{(4)}=$ 1.414 , n.s.) and an equivalent high level of freezing to the $\mathrm{T}$ stimulus 24 h later $\left(t_{(8)}=0.727\right.$, n.s.) (Fig. 2B). Thus, the loss of memory to the tone was due to the reactivation of the memory triggered by the presentation of the light stimulus. More importantly, when both T and L stimuli were conditioned to the same US separately (dual threat conditioning procedure: intermixed trials of four T-US and four L-US pairings, ITI of 1-4 min; see Fig. $2 \mathrm{C}$, top, for the schematic of the experimental procedures), instead of as the compound stimulus, intra-LA anisomycin infused after reactivation to the $\mathrm{L}$ stimulus did not interfere with the LTM to the T stimulus. Both ACSF $(n=6)$ and Aniso $(n=6)$ groups expressed a similar high level of freezing during the light reactivation $\left(t_{(10)}=0.576\right.$, n.s.), as well as during the tone LTM test $\left(t_{(10)}=\right.$ 1.896 , n.s.) (Fig. 2C). This pattern of results demonstrates that two distinct stimuli separately paired with the same US within the same training session can undergo reconsolidation independently of each other, thus confirming the high selectivity of the reconsolidation process we reported previously in our earlier studies using a similar dual threat conditioning protocol (Doyère et al. 2007; Debiec et al. 2010; Diaz-Mataix et al. 2011). More importantly, in this study we demonstrate that this high selectivity can be lost when the stimuli have been conditioned together as a compound stimulus.

In our earlier study (Debiec et al. 2006) using a second-order threat conditioning paradigm, we observed that the effects of reconsolidation, disruption, and extinction procedures paralleled each other. We found that disruption of reconsolidation of second-order stimulus attenuates defensive reactions to this stimulus leaving intact responding to the associated first-order stimulus. Similarly, extinguishing responding to a second-order stimulus reduces defensive reactions to this stimulus and has no effect on responding to the associated first-order stimulus. The question arises whether extinction and reconsolidation procedures could both unravel aspects of memory organization which would predict their sensitivity to disruption. To test for this possibility, we thus asked whether extinction of the light stimulus would also decrease freezing responses to the tone stimulus when conditioned as a compound, but not when conditioned separately in the dual conditioning paradigm. Adult Sprague-Dawley rats $(n=7)$ underwent compound conditioning procedure as described above, and then $24 \mathrm{~h}$ later were submitted to an extinction procedure with 28 light-alone presentations (ITI was $1-3 \mathrm{~min}$ ) in a single session (see Fig. 3A, top, for the schematic of the procedures). Freezing to the $\mathrm{L}$ stimulus significantly decreased over the session (beginning [Early] vs. end [Late], paired $t_{(6)}=28.444$, $P<0.001)$. Twenty-four hours later, memory to light and tone stimuli was tested in intermixed presentations (three trials

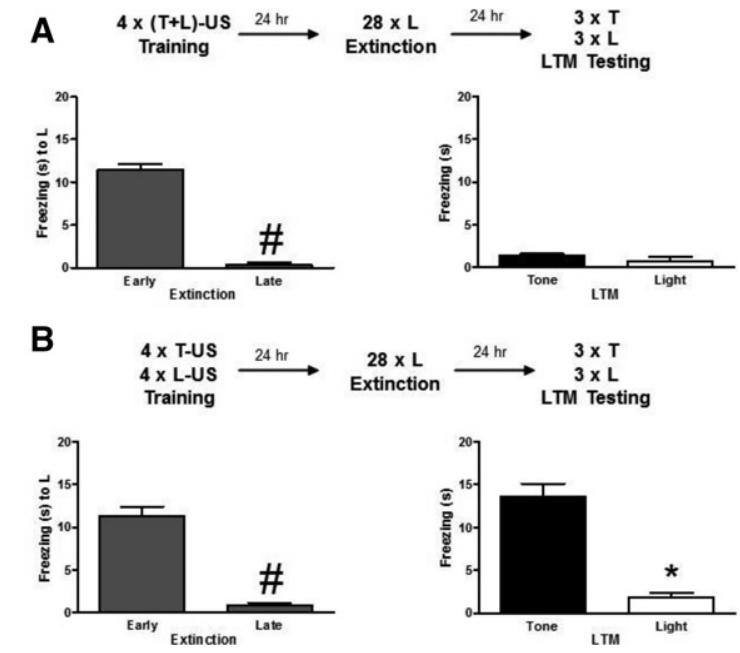

Figure 3. Extinction of one of the components of the compound stimulus induces the concomitant extinction of the other component. (A) Rats trained using a compound stimulus conditioning protocol and subjected $1 \mathrm{~d}$ later to 28 repeated presentations of the light component of the compound (extinction procedure) show decreased freezing to the tone during LTM test. Top panel: chart showing the experimental design. Left graph: freezing average (mean \pm SEM) across the first (Early) three and the last (Late) three light presentations during extinction. Right graph: mean \pm SEM freezing in response to the tone and the light averaged across stimuli presentations during LTM. (B) If light and tone were not presented as a compound during training but were paired individually with the US, the extinction of the light does not induce the extinction of the tone. Top panel: chart presenting the experimental design. Left graph: freezing average (mean \pm SEM) across the first three and the last three (out of 28) light presentations during extinction. Right graph: mean \pm SEM freezing in response to the tone and the light averaged across stimuli presentations during LTM. (T) Tone, (L) light, (US) unconditioned stimulus, (LTM) long-term memory, $\left({ }^{\#}\right) P<0.05$ vs. Early extinction, $\left({ }^{*}\right) P<0.05$ vs. Tone.

each). Animals showed equivalent low level of freezing to $\mathrm{L}$ and $\mathrm{T}$ stimuli (paired $t_{(6)}=1.113$, n.s.) (Fig. $2 \mathrm{~A}$ ). However, when rats were submitted to the dual conditioning paradigm $(n=8)$ (see Fig. 3B, top, for the outline of the procedures), extinction of $\mathrm{L}$ (beginning vs. end, paired $t_{(7)}=8.474, P<0.001$ ) resulted in a significant difference in the levels of freezing evoked by each of the two stimuli (light vs. tone [paired $t_{(7)}=9.909, P<0.001$ ]) (Fig. 3B). Thus, similar to the reconsolidation experiment, mediated extinction was observed when the stimuli were conditioned together as a compound, but not when they were conditioned in parallel (although sharing some conditioning parameters—same training session, same context, same US).

In our earlier studies we have demonstrated that memory reconsolidation may be cue-selective (Doyère et al. 2007) and that associatively reactivated memories are stable and resistant to disruption (Debiec et al. 2006). In contrast, the results of the current study show that if a light stimulus has been presented with the tone stimulus during a training session as a single compound stimulus, the retrieval of the light component triggers in the LA protein synthesis-dependent memory reconsolidation of the tone associated memory. These results are paralleled by our extinction experiments in which multiple presentations of the light stimulus, one of the components of the compound stimulus, extinguishes conditioned responding to the other component (the tone). One of the major differences between training conditions used in our previous studies demonstrating selectivity of reconsolidation in the amygdala and the current study is that selectivity of reconsolidation processes is observed when the stimuli were 
separated in time during conditioning, but not when the two cues are presented concomitantly. Temporal characteristics and relations between the stimuli do not only define associative learning (Balsam and Gallistel 2009), but also determine whether or not memories reconsolidate (Díaz-Mataix et al. 2013).Thus, the present data together with our previous reports suggest that specific encoding conditions control for the selectivity of the reconsolidation process: A loss of selectivity may be observed when a unique temporally bounded memory is formed.

This pattern of results raises a question about possible learning mechanisms that would account for these differential effects. One possibility is that in the compound conditioning protocol neural representations of two distinct events, tone and light, are encoded together in the amygdala either as a unique configure as posited by the configural theories of conditioning (for reviews, see Pearce 1994; Pearce and Bouton 2001), or as distinct (but not independent) elements individually linked to the same reinforcer as proposed by the elemental theories of conditioning (Rescorla and Wagner 1972; Pearce 1994). This would further determine that both components of the compound are retrieved and reconsolidated together even if only a single component is explicitly presented. The mediated-extinction effects (loss of responding to one component of the compound stimulus following extinction of the other component) we report here are in agreement with results obtained in previous studies using compound stimulus conditioning in other learning paradigms (Schnelker and Batsell 2006; Pineno 2007). According to the literature, depending on training conditions, the retrospective evaluation triggered by extinction will induce either mediated-extinction of the target stimulus or the opposite, recovery-from-overshadowing (i.e., increase of responding to one component following the extinction of the other). Parameters such as relative salience, simultaneous vs. serial stimulus presentations, and short or long duration of the compound stimulus may be of particular importance in controlling the effect (e.g., Shevill and Hall 2004; Liljeholm and Balleine 2006; Sissons et al. 2009). Therefore, it remains to be determined whether our paradigm favors symmetrical/bidirectional mediated-extinction, and thus, potentially, reconsolidation processes. It would also be of particular interest to determine whether reconsolidation processes would also be triggered in conditions that would favor recovery-from-overshadowing, or solely when the training conditions favor mediated-extinction, as the data on reconsolidation available so far may suggest (the present data and Debiec et al. [2006]).

Another question that remains to be resolved is whether visual events either alone or as a part of the compound are stored and reconsolidate in the LA. Although much of what we know about the role of the amygdala in threat conditioning comes from research using auditory cues, similar to auditory learning, the LA plays a critical role in the acquisition of visual threat conditioning (Campeau and Davis 1995; McDannald and Galarce 2011). Likewise, physiological studies of cellular responses in the LA in relation to conditioning have focused primarily on auditory stimuli (Bordi and LeDoux 1992; Romanski et al. 1993; Collins and Pare 2000; Repa et al. 2001; Goosens and Maren 2004; Humeau et al. 2007; An et al. 2012). However, some reports indicate that certain populations of cells in the LA respond both to visual and to auditory stimuli (Uwano et al. 1995; Toyomitsu et al. 2002; Nishijo et al. 2008). The question remains whether these multimodal cells are preferentially involved in encoding and storage of the compound stimulus conditioning, which would account for the lack of selectivity of reconsolidation processes observed in the present experiments. Another possibility is that in our paradigm visual events are stored outside of the amygdala (Sacco and Sacchetti 2010) or within a distinct nucleus of the amygdala. Despite the spatial separation, however, memories for these events would remain functionally connected with the amygdala-dependent elements of threat conditioning, such that when reactivated they render the amygdala-dependent memories labile.

In the current study, reconsolidation and extinction experiments paralleled each other in a way that in both cases an apparent loss of selectivity was observed when stimuli were conditioned in a compound conditioning paradigm. Interestingly, using second-order threat conditioning, we previously observed similar parallelism between reconsolidation and extinction (Debiec et al. 2006). Although extinction and reconsolidation are distinct processes involving different mechanisms (Duvarci and Nader 2004), such parallelism suggests that both processes have a similar target which, in turn, allows modification of the memory by combining reconsolidation and extinction procedures (Monfils et al. 2009). Using both reconsolidation and extinction procedures may thus help to uncover the intrinsic architecture of associations in the amygdala.

Both extinction and reconsolidation have been proposed as models of existing and novel treatments of anxiety disorders. The results of the present study suggest that when stimuli are temporally bound during memory formation, the fates of its components are intertwined. Thus, the way the memory is encoded is critical for its accessibility to modification, and determines boundary conditions for selective lability mechanisms and their use for effective treatments.

\section{Acknowledgments}

We thank Claudia Farb for her technical assistance. This research was supported by P50 MH058911 and R01 MH038774 grants to J.E.L. J.D. was supported by the 2010 NARSAD Young Investigator Award from the Brain \& Behavior Research Foundation. V.D. was supported by grants Memotime and TDE from Agence Nationale de la Recherche (ANR). V.D. and J.E.L. were recipients of collaborative grants from CNRS (CNRS-NYU-UPS) and from Partner University Fund (Emotion \& Time).

\section{References}

An B, Hong I, Choi S. 2012. Long-term neural correlates of reversible fear learning in the lateral amygdala. J Neurosci 32: 16845-16856.

Balsam PD, Gallistel CR. 2009. Temporal maps and informativeness in associative learning. Trends Neurosci 32: 73-78.

Bordi F, LeDoux J. 1992. Sensory tuning beyond the sensory system: An initial analysis of auditory response properties of neurons in the lateral amygdaloid nucleus and overlying areas of the striatum. J Neurosci 12: 2493-2503.

Campeau S, Davis M. 1995. Involvement of the central nucleus and basolateral complex of the amygdala in fear conditioning measured with fear-potentiated startle in rats trained concurrently with auditory and visual conditioned stimuli. J Neurosci 15: 2301-2311.

Collins DR, Pare D. 2000. Differential fear conditioning induces reciprocal changes in the sensory responses of lateral amygdala neurons to the $\mathrm{CS}^{+}$and $\mathrm{CS}^{-}$. Learn Mem 7: 97-103.

Debiec J, Doyere V, Nader K, LeDoux JE. 2006. Directly reactivated, but not indirectly reactivated, memories undergo reconsolidation in the amygdala. Proc Natl Acad Sci 103: 3428-3433.

Debiec J, Diaz-Mataix L, Bush DE, Doyere V, LeDoux JE. 2010. The amygdala encodes specific sensory features of an aversive reinforcer. Nat Neurosci 13: 536-537.

Debiec J, Bush DE, LeDoux JE. 2011. Noradrenergic enhancement of reconsolidation in the amygdala impairs extinction of conditioned fear in rats-a possible mechanism for the persistence of traumatic memories in PTSD. Depress Anxiety 28: 186-193.

Diaz-Mataix L, Debiec J, LeDoux JE, Doyere V. 2011. Sensory-specific associations stored in the lateral amygdala allow for selective alteration of fear memories. J Neurosci 31: 9538-9543.

Díaz-Mataix L, Ruiz Martinez RC, Schafe GE, LeDoux JE, Doyere V. 2013. Detection of a temporal error triggers reconsolidation of amygdaladependent memories. Curr Biol 23: 467-472. 
Doyère V, Debiec J, Monfils MH, Schafe GE, LeDoux JE. 2007. Synapsespecific reconsolidation of distinct fear memories in the lateral amygdala. Nat Neurosci 10: 414-416.

Dudai Y. 2012. The restless engram: Consolidations never end. Annu Rev Neurosci 35: 227-247.

Duvarci S, Nader K. 2004. Characterization of fear memory reconsolidation. J Neurosci 24: 9269-9275.

Eisenberg M, Kobilo T, Berman DE, Dudai Y. 2003. Stability of retrieved memory: Inverse correlation with trace dominance. Science 301: $1102-1104$.

Finnie PS, Nader K. 2012. The role of metaplasticity mechanisms in regulating memory destabilization and reconsolidation. Neurosci Biobehav Rev 36: 1667-1707.

Goosens KA, Maren S. 2004. NMDA receptors are essential for the acquisition, but not expression, of conditional fear and associative spike firing in the lateral amygdala. Eur J Neurosci 20: 537-548.

Humeau Y, Reisel D, Johnson AW, Borchardt T, Jensen V, Gebhardt C, Bosch V, Gass P, Bannerman DM, Good MA, et al. 2007. A pathway-specific function for different AMPA receptor subunits in amygdala long-term potentiation and fear conditioning. J Neurosci 27: 10947-10956.

Johansen JP, Cain CK, Ostroff LE, LeDoux JE. 2011. Molecular mechanisms of fear learning and memory. Cell 147: 509-524.

LeDoux J. 2012. Rethinking the emotional brain. Neuron 73: 653-676.

Lee JL. 2009. Reconsolidation: Maintaining memory relevance. Trends Neurosci 32: 413-420.

Liljeholm M, Balleine BW. 2006. Stimulus salience and retrospective revaluation. J Exp Psychol Anim Behav Process 32: 481-487.

Maren S. 2011. Seeking a spotless mind: Extinction, deconsolidation, and erasure of fear memory. Neuron 70: 830-845.

McDannald MA, Galarce EM. 2011. Measuring Pavlovian fear with conditioned freezing and conditioned suppression reveals different roles for the basolateral amygdala. Brain Res 1347: 82-89.

Milad MR, Quirk GJ. 2012. Fear extinction as a model for translational neuroscience: Ten years of progress. Annu Rev Psychol 63: 129-151.

Monfils MH, Cowansage KK, Klann E, LeDoux JE. 2009. Extinctionreconsolidation boundaries: Key to persistent attenuation of fear memories. Science 324: 951-955.

Myers KM, Davis M. 2007. Mechanisms of fear extinction. Mol Psychiatry 12: $120-150$

Nishijo H, Hori E, Tazumi T, Ono T. 2008. Neural correlates to both emotion and cognitive functions in the monkey amygdala. Behav Brain Res 188: $14-23$.

Paxinos G, Watson C. 1986. The rat brain in stereotaxic coordinates. Academic Press, San Diego, CA.

Pearce JM. 1994. Similarity and discrimination: A selective review and a connectionist model. Psychol Rev 101: 587-607.

Pearce JM, Bouton ME. 2001. Theories of associative learning in animals. Annu Rev Psychol 52: 111-139.
Pérez-Cuesta LM, Maldonado H. 2009. Memory reconsolidation and extinction in the crab: Mutual exclusion or coexistence? Learn Mem 16: $714-721$.

Pineno O. 2007. An examination of the effectiveness of inflation and deflation treatments in detecting within-compound learning of a taste aversion. Behav Processes 75: 33-39.

Repa JC, Muller J, Apergis J, Desrochers TM, Zhou Y, LeDoux JE. 2001. Two different lateral amygdala cell populations contribute to the initiation and storage of memory. Nat Neurosci 4: 724-731.

Rescorla RA, Wagner AR. 1972. A theory of Pavlovian conditioning: Variations in the effectiveness of reinforcement and nonreinforcement. In Classical conditioning II: Current research and theory (ed. Black AH, Prokasy WF), pp. 66-99. Appleton-Century-Crofts, New York.

Romanski LM, Clugnet MC, Bordi F, LeDoux JE. 1993. Somatosensory and auditory convergence in the lateral nucleus of the amygdala. Behav Neurosci 107: 444-450.

Runyan JD, Dash PK. 2005. Inhibition of hippocampal protein synthesis following recall disrupts expression of episodic-like memory in trace conditioning. Hippocampus 15: 333-339.

Sacco T, Sacchetti B. 2010. Role of secondary sensory cortices in emotional memory storage and retrieval in rats. Science 329: 649-656.

Schnelker J, Batsell WR Jr. 2006. Within-compound associations are not sufficient to produce taste-mediated odor potentiation. Behav Processes 73: $142-148$

Shevill I, Hall G. 2004. Retrospective revaluation effects in the conditioned suppression procedure. Q J Exp Psychol B 57: 331-347.

Sissons HT, Urcelay GP, Miller RR. 2009. Overshadowing and CS duration: Counteraction and a reexamination of the role of within-compound associations in cue competition. Learn Behav 37: 254-268.

Sotres-Bayon F, Diaz-Mataix L, Bush DE, LeDoux JE. 2009. Dissociable roles for the ventromedial prefrontal cortex and amygdala in fear extinction: NR2B contribution. Cereb Cortex 19: 474-482.

Stern SA, Alberini CM. 2013. Mechanisms of memory enhancement. Wiley Interdiscip Rev Syst Biol Med 5: 37-53.

Toyomitsu Y, Nishijo H, Uwano T, Kuratsu J, Ono T. 2002. Neuronal responses of the rat amygdala during extinction and reassociation learning in elementary and configural associative tasks. Eur J Neurosci 15: $753-768$.

Tronson NC, Taylor JR. 2007. Molecular mechanisms of memory reconsolidation. Nat Rev Neurosci 8: 262-275.

Uwano T, Nishijo H, Ono T, Tamura R. 1995. Neuronal responsiveness to various sensory stimuli, and associative learning in the rat amygdala. Neuroscience 68: 339-361.

Received April 22, 2013; accepted in revised form September 26, 2013. 


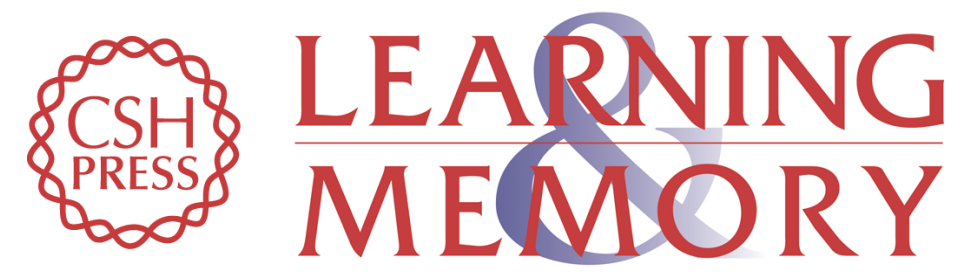

\section{The selectivity of aversive memory reconsolidation and extinction processes depends on the initial encoding of the Pavlovian association}

Jacek Debiec, Lorenzo Diaz-Mataix, David E.A. Bush, et al.

Learn. Mem. 2013, 20:

Access the most recent version at doi:10.1101//m.031609.113

References This article cites 43 articles, 12 of which can be accessed free at: http://learnmem.cshlp.org/content/20/12/695.full.html\#ref-list-1

Creative This article is distributed exclusively by Cold Spring Harbor Laboratory Press for the Commons first 12 months after the full-issue publication date (see

License http://learnmem.cshlp.org/site/misc/terms.xhtml). After 12 months, it is available under a Creative Commons License (Attribution-NonCommercial 3.0 Unported), as described at http://creativecommons.org/licenses/by-nc/3.0/.

Email Alerting Receive free email alerts when new articles cite this article - sign up in the box at the Service top right corner of the article or click here. 